\title{
The effect of spatial arrangement on judgments and errors in interpreting graphs
}

\author{
Cathleen McGrath ${ }^{a}$, Jim Blythe ${ }^{\mathrm{b}, \mathrm{l}}$, David Krackhardt ${ }^{\mathrm{a}}$ \\ ${ }^{\text {a } H e i n z ~ S c h o o l ~ o f ~ P u b l i c ~ P o l i c y ~ a n d ~ M a n a g e m e n t, ~ C a r n e g i e ~ M e l l o n ~ U n i v e r s i t y, ~} 5000$ Forbes Avenue, \\ Pittsburgh, PA 15213, USA \\ ${ }^{\mathrm{b}}$ School of Computer Science, Camegie Mellon University, Pittsburgh, PA, USA
}

\begin{abstract}
The spatial arrangement of social network data in graphs can influence viewers' perceptions of structural characteristics such as prominence, bridging and grouping. To study the extent of this effect, we conducted an experiment with 80 graduate students. Each student viewed three of five different spatial arrangements of the same network. We found that viewers' perceptions of structural features of the network changed as the spatial arrangement of the network changed. (C) 1997 Elsevier Science B.V.
\end{abstract}

Keywords: Spatial arrangements; Graphs; Perceptions; Experiment

\section{Introduction and problem statement}

It is commonly assumed that graphs communicate important characteristics of network data. In fact, the beginning of the field of social networks can be found in Moreno's use of the sociogram to communicate the power of network ideas (Moreno, 1953). As Harary points out, "It is very useful to have diagrams of graphs available for the accumulation of data leading to conjectures" (Harary, 1969, p. 213). When actors in networks are presented as nodes in a graph and the relationships between them are displayed as lines connecting the nodes, the graph conveys all the information contained in the adjacency matrix and so the two are 'informationally equivalent' (Larkin and Simon, 1987). However, the graph can also use Euclidean spatial relations to highlight the relationships among actors. Thus, drawing network data in a graph may influence individuals' perceptions of social network attributes completely determined by the structure of the network, such as the existence of subgroups and the relative centrality of actors.

\footnotetext{
${ }^{1}$ Also at 5000 Forbes Avenue, Pittsburgh, PA 15213, USA.
} 
In this paper we explore the influence of the spatial arrangement of networks on individuals' perceptions of common social network measures, in particular 'prominence' and 'bridging', which are two facets of centrality, and grouping. Prominence deals with a node's popularity and most closely relates to the degree of the node. Importance as a bridge deals with a node's strategic positioning between groups, and most closely relates to betweenness centrality (Freeman, 1978). By spatial arrangement we mean a mapping from the nodes of the graph to $\mathscr{R} \times \mathscr{R}$ (the real plane).

A large body of work exists on computer programs to draw graphs according to certain 'aesthetics' (see Di Battista et al., 1994, for a survey of this work). However, almost all of this work considers aesthetics that attempt to improve graph readability from a very general point of view without considering specific applications, and uses general aesthetics such as the regular spacing of nodes and minimization of edge crossings.

Only a few studies explicitly question the aesthetics in use and there has been very little work on analyzing how well they actually improve the information conveyed in a graph's spatial arrangement. Ding and Mateti (1990) consider the subjective factors that go into the drawing of a diagram intended to explain data structures in computer programs. They produce their factors by examining the pictures that appear in a number of text books in that field. Batini et al. (1985) make an experimental study of the aesthetics used in entity relationship diagrams from the field of software engineering. We are not aware of any studies that consider the information one might want to convey in a social network spatial arrangement.

In this paper we present an empirical study of how the spatial positioning of nodes in the graph influences individuals' perceptions of network characteristics. We build on previous work on graph drawing and apply it specifically to drawing graphs for social networks. We find that individuals' perceptions of graphs may depend on both the structure of the network itself, that is the pattern of ties between nodes, and the spatial arrangement of nodes.

In the rest of this section we describe the graph theoretical and Euclidean features of a spatial arrangement that might influence the values an individual assigns to measures of prominence, bridging, and grouping while looking at a graph. In the subsequent sections we describe our experimental design and the results of the study.

Our experiment was conducted using five different spatial arrangements of one particular network having twelve actors and 48 ties. This framework allows us to hold structural relationships among nodes constant while varying their spatial relationships. The arrangements vary in the proximity of nodes to each other and the positioning of nodes toward the center or periphery of the graph.

The factors that influence perception of graphs can be divided into two groups: those that are concerned with spatial properties of the graph layout and those that are concerned with graph theoretical properties of the network itself. In this study we consider how Euclidean spatial factors affect the viewer's perception of the graph when structural features are held constant. In particular we consider the influence of:

1. proximity to the center of spatial arrangement on perception of prominence;

2. positioning between clusters of nodes in spatial arrangement on perception of bridging; 
3. spatial clustering of groups of nodes in spatial arrangement on perception of grouping.

In Section 3 we explain in more detail how the various measures were calculated and show our results based on ordinary least squares (OLS) and analysis of errors in reporting.

\section{Experimental design}

\subsection{The study}

Eighty graduate students who had just completed a course in organizational theory (a course that emphasized the importance of understanding networks in organizations) volunteered to be subjects in the experiment. The subjects were given a questionnaire containing three of the five graph spatial arrangements and told the following:

The following three graphs are modeled after networks of communications observed in three different merger and acquisition teams of an investment banking firm. A connection between two team members means that they discuss work-related matters with each other. If no line exists between two team members then they never discuss work with each other.

All nodes were labeled with first names in the arrangements presented to the subjects. By providing a context, the investment banking firm and member names, we attempt to focus the subjects' attention on the social aspect of communication networks. In every arrangement, each node was mapped to a new name. For analysis and discussion purposes, we have relabeled the nodes of interest with letters from $A$ to $E$. For prominence and bridging, $\mathrm{A}$ and $\mathrm{B}$ have the highest scores, followed by $\mathrm{C}$, and $\mathrm{D}$ and $\mathrm{E}$ have the lowest scores. The five spatial arrangements are shown in Fig. 1. It is important to remember that all five spatial arrangements display identical networks.

We asked respondents two questions about the same five focal nodes in each graph: how 'prominent' was each of five particular players in the graph; and how important a 'bridging' role did each of these five particular players occupy in the graph. We also asked them to report the number of subgroups in the graph. Subjects rated the prominence and bridging of the same five nodes (with different first name labels) for each spatial arrangement by circling a number from 1 to 7 on a Likert scale that went from no: prominent (or not important as a bridge) to most prominent (or most important as a bridge). The question about prominence was worded as follows: "Some individuals have a more prominent role in their team than other individuals. Please rate the following people according to how prominent within their team they appear to you by circling the appropriate number next to each name." The question about importance as a bridge was worded in the following way: "Some individuals are important because they form a bridge between subgroups. Please rate the following people according to how important they appear to be as bridges between subgroups by circling the appropriate number next to each name." Finally, we created the questionnaire using a format that allowed one spatial arrangement and questions about that spatial arrangement to be viewed at the same time, without viewing any other spatial arrangement. 

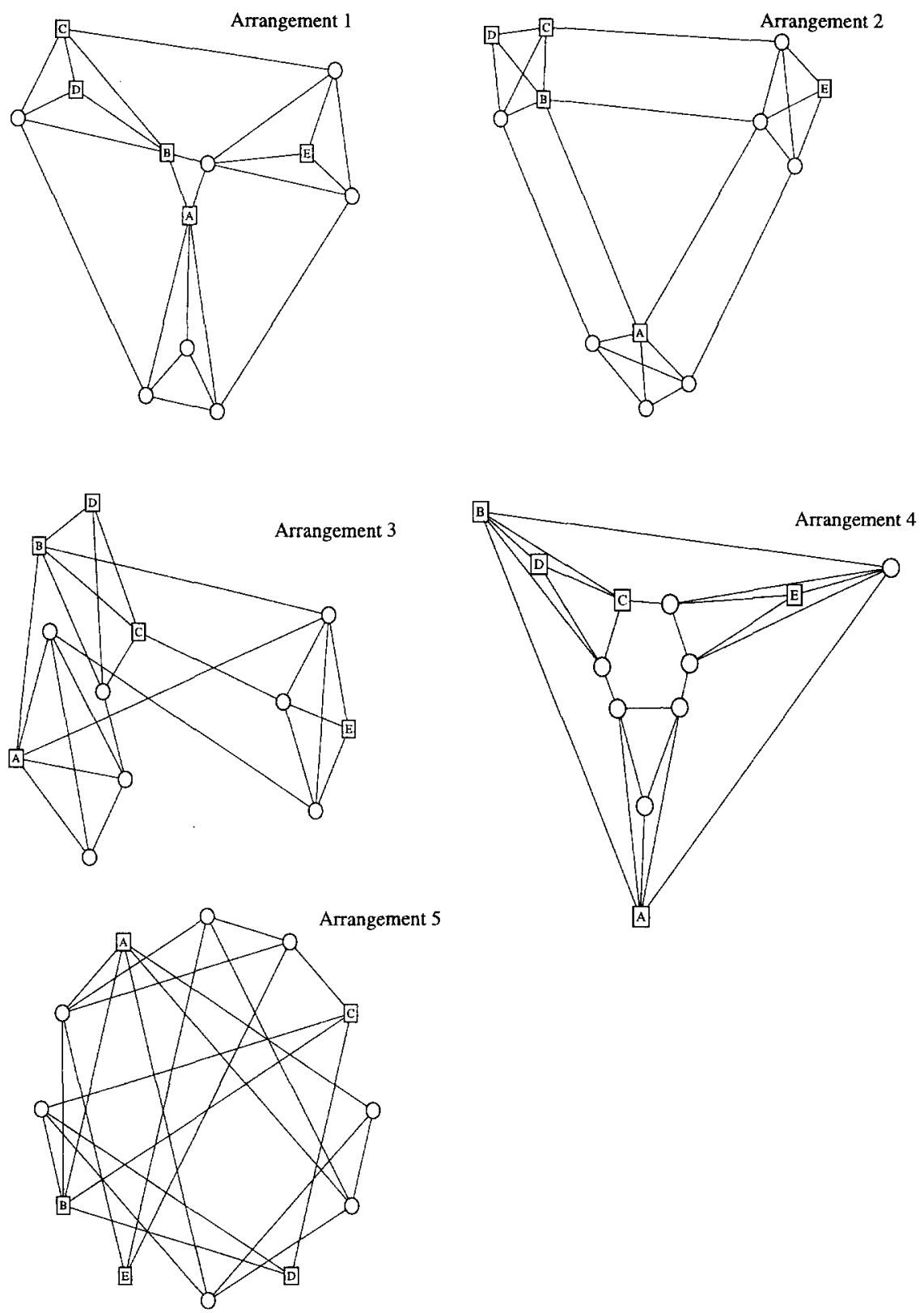

Fig. 1. The five different spatial arrangements of the same labeled graph.

We used an incomplete balanced Latin square design to control for order in the presentation of the graphs by displaying each spatial arrangement first, second, and third exactly once. There were five versions of the questionnaire, each version containing only three of the five drawings. Table 1 depicts which questionnaire version (Q1 to Q5) 
Table 1

Order of appearance of spatial arrangements in five questionnaires

\begin{tabular}{lllllll}
\hline & A1 & A2 & A3 & A4 & A5 & $N$ \\
\hline Q1 & 1 & & 2 & & 3 & 14 \\
Q2 & & 2 & 1 & 3 & & 18 \\
Q3 & & & 3 & 2 & 1 & 16 \\
Q4 & 3 & 1 & & & 2 & 20 \\
Q5 & 2 & 3 & & 1 & & 12 \\
\hline
\end{tabular}

contained which spatial arrangements (A1 to A5) and the order in which those spatial arrangements occurred. For example, the second row (labeled ' $Q 2$ ' in Table 1) indicates that Questionnaire 2 presented the respondent with Arrangement 3 (A3) first, followed by Arrangement 2, followed by Arrangement 4 . By design, each spatial arrangement is preceded by every other except one exactly once. Order effects are discusses in Section 3.1 .

\subsection{The network}

The network itself is symmetric, with twelve actors and 48 directed ties. We used a small graph so that the subjects would not be overwhelmed by the amount of information presented to them. The overall density is $36 \%$. Table 2 shows values for the two measures of centrality for nodes A through E. Degree centrality measures the number of nodes that are adjacent to the focal node. Betweenness centrality measures the number of times a node is on the shortest path between two other nodes (Freeman, 1978). In our experiment, degree centrality and betweenness centrality are strongly correlated $(r=$ 0.93). While degree centrality and betweenness centrality remain conceptually distinct, in this case they are not very different empirically. Nodes A and B are automorphically equivalent as are nodes D and E (see Borgatti and Everett, 1992, for a discussion of automorphic equivalence). The network has four cliques.

Fig. 1 shows the five different spatial arrangements in which the network was presented. Keeping the aesthetics standards of regular spacing of nodes and minimization of edge crossings in mind, we arranged the graph in ways that would be interpretable by the respondents. Arrangement 5 , the circle, was not drawn with the

Table 2

Centrality measures

\begin{tabular}{lll}
\hline & Degree centrality & Freeman betweenness centrality \\
\hline A & 5 & 8.67 \\
B & 5 & 8.67 \\
C & 4 & 4.67 \\
D & 3 & 0.00 \\
E & 3 & 0.00 \\
\hline
\end{tabular}


standards in mind. It is included because of its general acceptance as a means for presenting social network data. In his handbook on social network analysis Scott says: various ad hoc extensions to the idea of the sociogram have been used as researchers have sought to complement their mathematical measures with some kind of diagrammatic representations. One common technique has been to construct the sociogram around the circumference of a circle so that the pattern of lines becomes more visible... (Scott, 1991, p. 148).

\section{Results}

\subsection{Order effects}

Because each subject provided responses for three spatial arrangements, we compare both within and between subjects. We were concerned that the within subject comparisons may be biased if subjects were learning about interpreting graphs as they moved from the first to the third graph evaluation task. Poulton (1982) discusses the strategy transfer hypothesis describing cases in which subjects learn a strategy in one condition and transfer it to another condition. In exploring the effect of order, we found that there were no significant overall mean effects. To test for order effects, we ran two regressions, one with prominence as the dependent variable and one with bridging as the dependent variable. In each regression we controlled for individual respondents, the node, and the layout. When we included order of appearance, order had no significant effect on the independent variable for either prominence or bridging.

However, when each spatial arrangement was considered separately, Arrangement 3 showed some difference depending on order, as seen in Table 3. This suggests that Arrangement 3 may be particularly difficult to interpret and therefore subject to different interpretations depending on the layout after which it appears. None of the other spatial arrangements showed any significant difference in prominence scores when separated by order of appearance.

\subsection{Predicting judgment}

The results of our analysis of individuals' reports of each node's prominence and importance as a bridge and of the overall number of groups in the network suggest that spatial relationships between nodes influence viewers' perceptions of the graphs. The

Table 3

Analysis of variance for mean centered prominence scores for Arrangement 3 (the only arrangement showing significant order effects for prominence)

\begin{tabular}{lrrrl}
\hline & \multicolumn{1}{c}{ First } & Second & Third & $F$ value \\
\hline A & 1.58 & 1.01 & 0.57 & 2.76 \\
B & 1.77 & 0.70 & 0.31 & $4.91^{* *}$ \\
C & 0.33 & -0.22 & 0.51 & $1.98^{* *}$ \\
D & -2.29 & -0.53 & -0.56 & $8.68^{* *}$ \\
E & -2.23 & -0.61 & -0.49 & $7.66^{* * *}$ \\
\hline
\end{tabular}

${ }^{*}{ }^{*} p<0.01 ;{ }^{* * *} p<0.001$. 
Table 4

Individual mean centered scores for prominence (standard deviation in parentheses)

\begin{tabular}{lccccc}
\hline & Arrangement 1 & Arrangement 2 & Arrangement 3 & Arrangement 4 & Arrangement 5 \\
\hline A & $0.69(1.79)$ & $1.46(1.18)$ & $1.11(1.24)$ & $1.08(1.60)$ & $0.36(1.88)$ \\
B & $0.64(1.86)$ & $1.30(1.39)$ & $1.00(1.45)$ & $1.06(1.59)$ & $0.29(1.76)$ \\
C & $-0.30(1.30)$ & $-0.13(0.92)$ & $0.24(1.00)$ & $0.26(1.42)$ & $-0.42(1.60)$ \\
D & $-0.12(1.39)$ & $-0.96(1.29)$ & $-1.15(1.54)$ & $-0.24(1.47)$ & $-0.93(1.32)$ \\
E & $-0.16(1.67)$ & $-1.58(1.31)$ & $-1.18(1.55)$ & $-0.40(1.48)$ & $-1.02(1.64)$ \\
\hline
\end{tabular}

mean centered average prominence and importance as a bridge for each node in every arrangement shows that while the ordering of prominence and bridging does not change across spatial arrangements, the relative values assigned to prominence and bridging change. We use OLS to compare Arrangements 1 through 4 to Arrangement 5, the circle. When nodes are arranged in a circle, they are all equally placed from the center and equally placed between other nodes. There is no variation in the spatial information provided in the circle.

For grouping, we compare the distribution of groups across all five layouts and find some differences in the shapes of the distributions across the five layouts. The following sections give details of the analysis for prominence, importance as a bridge, and grouping.

\subsection{J. Prominence}

Before reporting the prominence data, we converted the prominence reported on a seven-point Likert scale to a mean centered prominence score. This controls for individuals' tendencies to rate high or low in general. We did this by subtracting each respondent's average prominence score across all three spatial arrangements from the prominence score assigned to the node by the respondent. The average mean centered prominence scores for each node in every spatial arrangement are reported in Table 4. Overall, the ordering of the value for prominence is stable. Ordering changes only once, in Arrangement 1, where node C's mean centered average prominence is less than node D's and node E's.

Next, we estimate the prominence for each node using arrangements as the indepen-

Table 5

Regression prominence by node

\begin{tabular}{lccccc}
\hline Variable & $\mathrm{A}$ & $\mathrm{B}$ & $\mathrm{C}$ & $\mathrm{D}$ & \multicolumn{1}{c}{$\mathrm{E}$} \\
\hline Inter & $4.32^{* * *}$ & $4.48^{* * *}$ & $3.05^{* * *}$ & $2.92^{* * *}$ & $2.80^{* * *}$ \\
$\mathrm{~A} 1$ & $0.21^{* *}$ & 0.05 & $-0.12^{*}$ & $0.70^{*}$ & $0.93^{* *}$ \\
$\mathrm{~A} 2$ & $0.83^{*}$ & 0.50 & -0.03 & -0.46 & -0.33 \\
$\mathrm{~A} 3$ & $0.69^{*}$ & 0.42 & 0.48 & -0.16 & -0.09 \\
$\mathrm{~A} 4$ & 0.50 & 0.21 & 0.26 & $0.72^{*}$ & $0.72^{*}$ \\
$R^{2}$ & 0.423 & $0.459^{*}$ & 0.340 & $0.587^{* * *}$ & $0.593^{* * *}$ \\
$N$ & 232 & $232^{*}$ & 229 & 229 & $232^{*}$ \\
$R^{2 *}$ (ID control only) & 0.391 & $0.446^{*}$ & 0.319 & $0.517^{* * *}$ & $0.520^{* * *}$ \\
$R^{2}-R^{2 *}$ & 0.031 & $0.011^{* * *}$ & 0.021 & $0.070^{* *}$ & $0.072^{* * *}$ \\
\hline
\end{tabular}

${ }^{*} p<0.05 ;^{* *} p<0.01{ }^{* * *} p<0.001$. 
dent variable. The results are shown in Table 5. We include individual identifiers to control for individuals' tendency to rate high or low. And we test for the joint significance of the arrangement variables by comparing $R^{2}$ for an equation containing the identifier controls and the arrangement variables $\left(R^{2}\right)$ to the $R^{2}$ for an equation containing only the identifier variables $\left(R^{2 *}\right)$. Nodes $\mathrm{D}$ and $\mathrm{E}$ are the only nodes for which the arrangement variables are jointly significant. $\mathrm{D}$ and $\mathrm{E}$ may be most amenable to the influence of arrangement because they are the least structurally prominent.

Node $\mathrm{C}$ follows the expected pattern, that is, in Arrangement 1 and Arrangement 2, when $\mathbf{C}$ is on the periphery, its coefficients predict values for prominence that are less than the circle. In Arrangements 3 and 4, when $C$ moves toward the center of the graph, its coefficients become positive. Similarly, nodes D and $\mathrm{E}$ have positive coefficients for Arrangements 1 and 4 when they are closer to the center of the graph, and negative coefficients for Arrangements 2 and 3 when they are more peripheral. Nodes A and B provide a contradiction to the simple spatial theory we propose. They are not significantly more prominent in Arrangement 1 where they are members of a central cluster. This result contradicts the proposition that positioning in the center of the graph enhances the perceived prominence of nodes. One explanation for this might be that nodes with high structural prominence are not affected by spatial positioning as much as nodes with low structural prominence.

\subsubsection{Importance as a bridge}

Table 6 shows the mean centered bridging scores for all five nodes and five arrangements. The order of reported bridging is consistent for all nodes in all arrangements: $A$ and $B$ are always greater than $C$ which is always greater than $D$ and $E$.

Again, we estimate each node's importance as a bridge using arrangements as the independent variable, including individual identifiers and testing for joint significance. The results are shown in Table 7 . For bridging, the arrangement variables are jointly significant for nodes $\mathrm{A}, \mathrm{B}$ and $\mathrm{E}$.

Both A and B have higher bridging scores in Arrangements 1 and 2 when they are positioned between two clusters than they do in Arrangements 3 and 4 when they are not. Node $\mathrm{C}$ follows a similar pattern. In Arrangements 1 and 2, $\mathrm{C}$ is not the clique member closest to another clique and its reported bridging is lower than in Arrangements 3 and 4 , when $C$ is positioned between clusters. $D$ and $E$ are reported to be less important as a bridge in Arrangements 1 through 4 than they are in the circle. However, the coefficients for $\mathrm{E}$ are not statistically significant.

Positioning nodes between groups seems to enhance the perception of their impor-

Table 6

Individual mean centered scores for importance as bridge (standard deviation in parentheses)

\begin{tabular}{lccccc}
\hline & Arrangement 1 & Arrangement 2 & Arrangement 3 & Arrangement 4 & Arrangement 5 \\
\hline A & $1.73(1.45)$ & $2.02(0.91)$ & $1.51(1.29)$ & $1.30(1.82)$ & $0.68(1.66)$ \\
B & $1.76(1.41)$ & $1.88(1.25)$ & $1.53(1.16)$ & $1.47(1.64)$ & $0.80(1.60)$ \\
C & $0.27(1.24)$ & $0.24(1.02)$ & $0.53(1.25)$ & $0.84(1.37)$ & $-0.47(1.52)$ \\
D & $-1.74(1.33)$ & $-2.06(0.99)$ & $-1.75(1.16)$ & $-1.35(1.67)$ & $-0.86(1.44)$ \\
E & $-1.63(1.48)$ & $-2.10(0.96)$ & $-1.70(1.39)$ & $-1.60(1.42)$ & $-1.09(1.80)$ \\
\hline
\end{tabular}


Table 7

Regression bridging by node

\begin{tabular}{|c|c|c|c|c|c|}
\hline Variable & A & B & $\mathrm{C}$ & $\mathrm{D}$ & $\mathrm{E}$ \\
\hline Inter & $3.95^{* * *}$ & $3.94 * * *$ & $2.71 * * *$ & $3.57^{* * *}$ & $3.34^{* * *}$ \\
\hline A1 & $0.89^{* *}$ & $1.07 * * *$ & 0.46 & $-0.90^{* * *}$ & -0.48 \\
\hline $\mathrm{A} 2$ & $1.26^{* * *}$ & $1.12^{* * *}$ & 0.40 & $-0.80^{* *}$ & -0.54 \\
\hline A3 & $0.69^{*}$ & $0.89^{* *}$ & $0.78 * *$ & $-0.64^{*}$ & -0.36 \\
\hline A4 & 0.55 & $0.65^{*}$ & $0.75^{*}$ & -0.31 & -0.18 \\
\hline$R^{2}$ & $0.576^{* * *}$ & $0.550^{* * *}$ & $0.468^{* *}$ & $0.642^{* * *}$ & $0.560 * * *$ \\
\hline$N$ & 230 & 232 & 231 & 231 & 233 \\
\hline$R^{2 *}$ (ID control only) & $0.514^{* * *}$ & $0.484 * * *$ & $0.437^{*}$ & $0.598^{* * *}$ & $0.545^{* * *}$ \\
\hline$R^{2}-R^{2 *}$ & $0.061^{* * *}$ & $0.066^{* * *}$ & 0.032 & $0.044^{* * *}$ & 0.014 \\
\hline
\end{tabular}

tance as a bridge. And similarly, positioning toward the center of the graph seems to highlight prominence. For both prominence and bridging we see that spatial positioning can draw attention to particular characteristics of the network and therefore influence viewers' perceptions of the network.

\subsubsection{Grouping}

The number of subgroups that individuals perceive in a graph may be influenced by the spatial clustering of nodes in the arrangement. Spatial clustering of nodes may influence judgment of the presence of groups independent of the structure of ties between the nodes. Table 8 shows the mean number of groups reported for each spatial arrangement. An analysis of variance of the reported number of groups reported for each spatial arrangement showed that there was a significant difference between spatial arrangements at the 0.08 level.

We also consider the shape of the distributions of the number of groups reported. Fig. 2 shows the distribution of responses for the number of groups for all five spatial arrangements. Here we see that Arrangement 5, the circle, has a distribution that is much flatter than the rest, suggesting that respondents could not determine groupings with consistency in the circle spatial arrangement. Arrangement 3, which presented the nodes clumped in two groups, one large and one small, was not interpreted as two groups by many people. We used the Kolmogorov-Smirnov test to determine if each pair of distributions of reported number of groups could be considered statistically significantly different. The results in Table 9 show that the distribution of number of groups for Arrangement 5 differs from the distribution of number of groups for every other spatial

Table 8

Mean number of groups reported in each spatial arrangement (standard deviation in parentheses)

\begin{tabular}{ll}
\hline Arrangement & Mean number of groups \\
\hline 1 & $5.04(3.42)$ \\
2 & $4.27(3.70)$ \\
3 & $3.55(1.59)$ \\
4 & $4.75(3.90)$ \\
5 & $3.45(2.90)$ \\
\hline
\end{tabular}




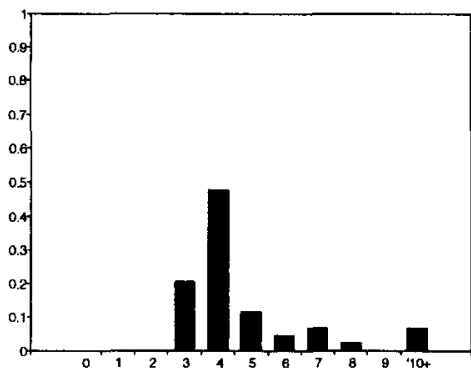

Arrangement 1

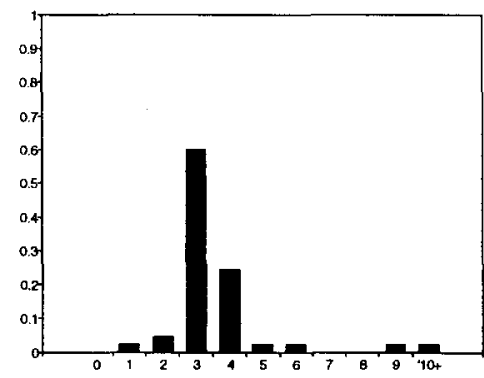

Arrangement 3

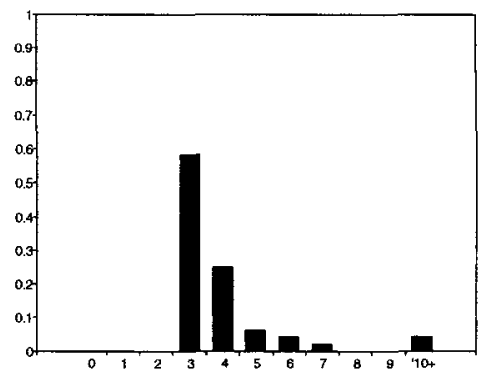

Arrangement 2

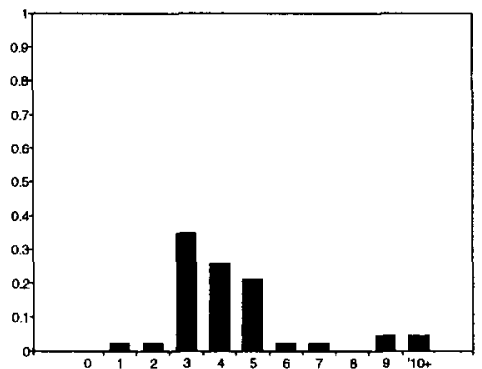

Arrangement 4

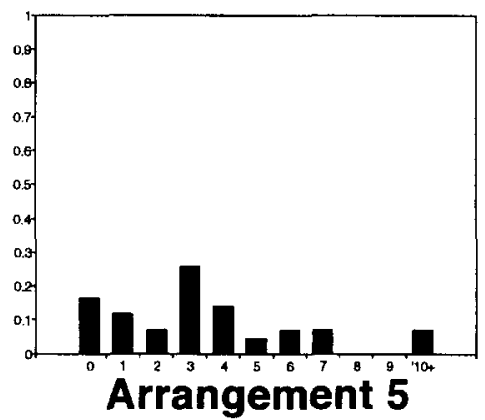

Fig. 2. Distributions of the number of groups reported for each spatial arrangement.

arrangement. Arrangement 1 differs from Arrangements 2 and 3 as well. Fig. 3 shows the multidimensional scaling of the difference in distribution of number of groups for all five spatial arrangements. A line between two spatial arrangements shows that the Table 9

Kolmogorov-Smimov test for difference of distribution of number of groups ( $p$ value in parentheses)

\begin{tabular}{llllll}
\hline & Arrangement 1 & Arrangement 2 & Arrangement 3 & Arrangement 4 & Arrangement 5 \\
\hline A1 & $\mathrm{X}$ & $0.38(0.003)$ & $0.46(0.001)$ & $0.19(0.41)$ & $0.40(0.002)$ \\
A2 & & $\mathrm{X}$ & $0.08(0.99)$ & $0.19(0.40)$ & $0.35(0.008)$ \\
A3 & & $\mathrm{X}$ & $0.27(0.08)$ & $0.28(0.06)$ \\
A4 & & & $\mathrm{X}$ & $0.30(0.04)$ \\
A5 & & & & $\mathrm{X}$ \\
\hline
\end{tabular}


A5

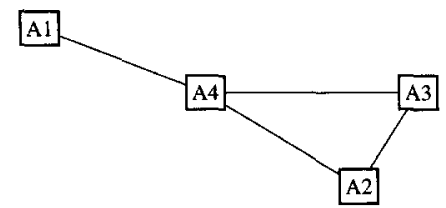

Fig. 3. Multidimensional scaling of difference of distributions in number of groups attributed to the graph. Lines between nodes indicate no statistically significant difference in their distributions.

Kolmogorov-Smirnov test did not show a statistically significant difference in the distribution of the number of reported groups between the two spatial arrangements.

\subsection{Drawing 'good pictures'}

It is probably not possible to say that one spatial arrangement is the 'best' spatial arrangement of a particular network. Often the best spatial arrangement is the one that highlights the characteristic of the network that is being discussed, and there may be no single arrangement that best highlights every characteristic. For the network we studied, however, it could be argued that a 'good' spatial arrangement is one that highlights the prominence and bridging for the five nodes of interest, and clearly displays the group structure.

In the graph used in this study, it is clear, from Table 2 that, structurally, nodes $A$ and $B$ are the most prominent nodes, followed by node $C$, and that nodes $D$ and $E$ are the least prominent. The same ordering holds for bridging: nodes $\mathrm{A}$ and $\mathrm{B}$ are highest in betweenness centrality, followed by $\mathrm{C}$, followed by $\mathrm{D}$ and $\mathrm{E}$. A minimum standard, then, for a spatial arrangement is one that clearly communicates the order of these nodes on such structural features. To determine the relative merits of our five arrangements, we compare the number of times respondents report this ordering of nodes based on their judgments of prominence and bridging in each spatial arrangement. Fig. 4 shows proportion of ordering for bridging and prominence that are 'correct', by which we mean, respondents reported the following order: $A=B>C>D=E$. Arrangement 2 has the highest proportion of correctly ordered responses for both prominence and bridging. Arrangements 1 and 4 have the next highest proportion of correctly ordered responses, followed by Arrangement 3 and finally Arrangement 5. This suggests that Arrangement 2 is the 'best' spatial arrangement to convey prominence and bridging. On the other hand, Fig. 2 shows that Arrangement 1 has the highest proportion of correct responses for the number of groups, four, in the network.

To understand better what sort of errors occurred in reporting prominence and bridging, we separated the pair-wise orderings into their three logical groupings: for any pair of nodes $i$ and $j, i>j, i=j$, or $i<j$, on either structural dimension. Tables A.1 to A.10 in Appendix A show the number of times each ordered pair occurs with the number of correctly ordered pairs highlighted in boldface. It is interesting to note that respondents' judgments were better overall for bridging than for prominence. Table A.1 


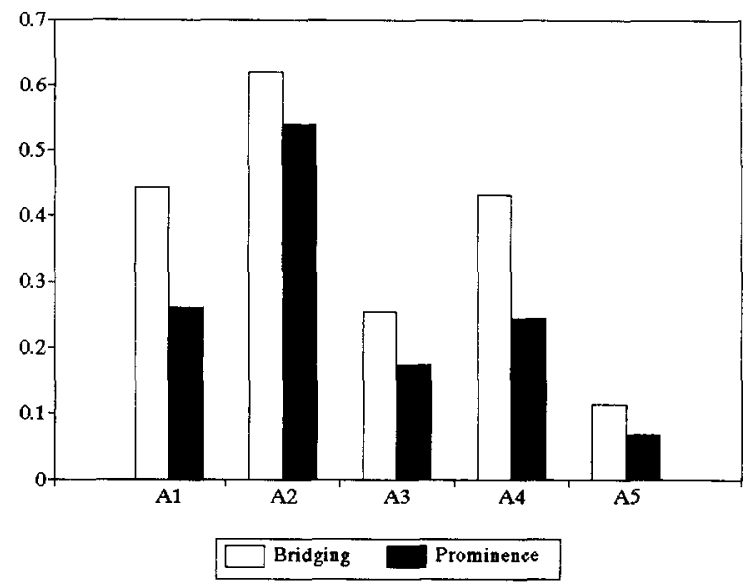

Fig. 4. The proportion of responses with correct ordering of nodes for each arrangement.

in Appendix A shows that $47.7 \%$ of the respondents recognized that nodes $\mathrm{A}$ and $\mathrm{B}$ were equally prominent and that $40.9 \%$ of the respondents judged node $\mathrm{C}$ to be less prominent than node $\mathrm{E}$ even though node $\mathrm{C}$ is truly more prominent than node $\mathrm{E}$. For bridging, Table A. 6 shows that $70.5 \%$ of the respondents recognized that nodes $\mathrm{A}$ and $\mathrm{B}$ were equally important as a bridge and only $13.6 \%$ of respondents judged $\mathrm{C}$ to be less important as a bridge than node E. Table A.2 shows that almost one third of the respondents judged nodes $B$ and $C$ to be equal, although $B$ is truly more prominent than $\mathrm{C}$; again only $14.3 \%$ make this error for bridging (although $\mathrm{B}$ and $\mathrm{C}$ are equally on the interface of their spatial cluster).

In order to present concise information about the tables of pair-wise comparisons of the observed order of nodes, we define three types of error: an $\alpha$ error occurs when a pair of nodes are the same on the structural attribute (such as nodes A and B are on betweenness) but are judged as different on that attribute by the respondent; a $\boldsymbol{\beta}$ error occurs when a pair of nodes are different on a structural attribute (such as B and $\mathrm{C}$ are on betweenness) but are judged as the same on that attribute by the respondent; a $\gamma$ error occurs when a pair of nodes are different on a structural attribute (as C and D are on betweenness) but are judged in reverse order from their true order on that attribute by the respondent.

The comparisons of the errors broken down into these types are shown in Figs. 5 and 6 for prominence and bridging respectively. Overall, respondents had lower error rates for bridging than for prominence. The circle had relatively high $\beta$ error for bridging (and to a less extent prominence) compared to the other spatial arrangements. This suggests that a circular representation tends to hide differences between nodes. Looking at the reported errors, we see that except for the circle, there is much less $\beta$ error (reporting nodes equal when they are not) for bridging than there is for prominence. Fig. 5 shows a relatively high $\gamma$ error rate for Arrangement 1 . That means that many people reversed the order of nodes' prominence when looking at Arrangement 1.

The observation that the circle has a relatively low $\gamma$ error rate led us to another interesting way to consider the quality of spatial arrangements. The low $\gamma$ error rate for 


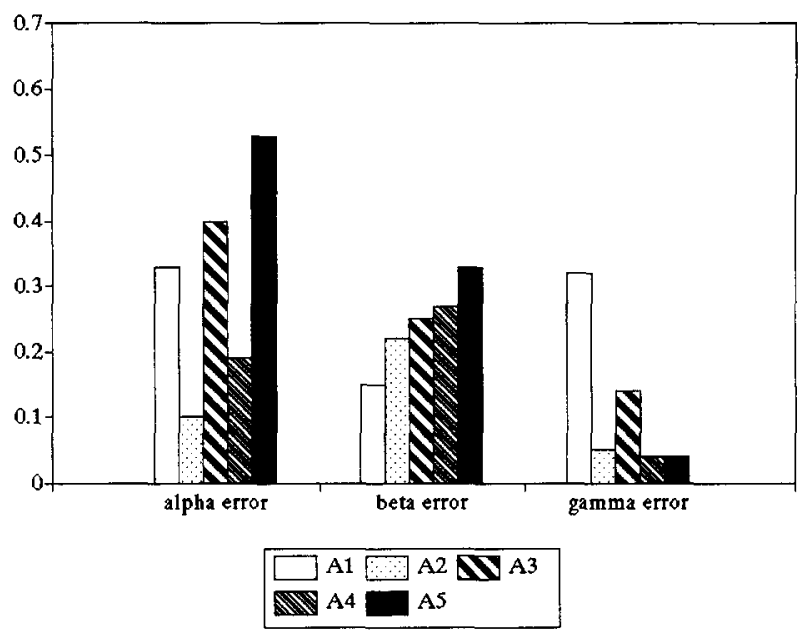

Fig. 5. Percent error for prominence: $\alpha=$ nodes truly the same but judged as different; $\beta=$ nodes truly different but judged as the same; $\gamma=$ nodes truly different but judged in reverse order from their true order.

Arrangement 5 can be explained by the unusually high incidence of respondents' reporting that all nodes are equally prominent or equally important as a bridge for Arrangement 5. When the respondent assigns all nodes equal values, a $\gamma$ error is not possible. In general, 'good spatial arrangements' should not leave the viewer with the impression that all nodes have the same values for prominence or bridging when in fact they vary on these dimensions.

Our final consideration of error in reporting will show the proportion of responses in which all nodes were assigned the same value. As Table 10 shows for both prominence and bridging, Arrangement 5 has a much higher incidence of respondents' reporting that

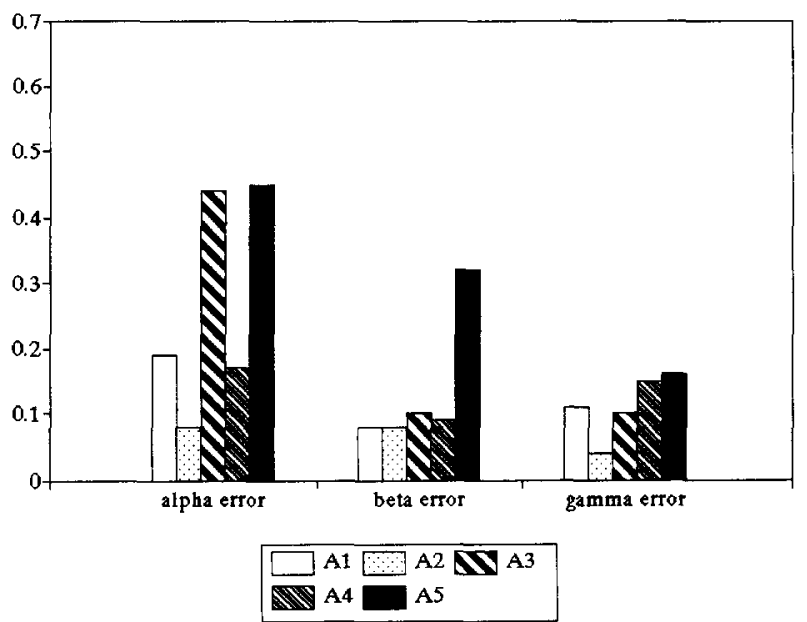

Fig. 6. Percent error for bridging: $\alpha=$ nodes truly the same but judged as different; $\beta=$ nodes truly different but judged as the same; $\gamma=$ nodes truly different but judged in reverse order from their true order. 
Table 10

Proportion of respondents who assigned every node in a given arrangement the same prominence or bridging score ( $N$ in parentheses)

\begin{tabular}{lll}
\hline & Prominence & Bridging \\
\hline A1 & $0.04(46)$ & $0.00(46)$ \\
A2 & $0.18(50)$ & $0.02(50)$ \\
A3 & $0.15(48)$ & $0.04(48)$ \\
A4 & $0.17(46)$ & $0.04(46)$ \\
A5 & $0.24(50)$ & $0.28(50)$ \\
\hline
\end{tabular}

all nodes are equal than any other spatial arrangement. This is especially true for bridging, where a full $29 \%$ of the respondents see no differences among the five nodes in the circle with regard to bridging, while each other arrangement had at most $4 \%$ of the respondents give equal bridging scores to all five nodes.

\section{Conclusions}

We have shown that spatial arrangement has a significant impact on viewers' judgments of prominence, bridging and grouping. We conducted the first empirical study of the influence of the arrangement on these judgments, and while it is dangerous to draw strong conclusions from this small experiment, there is evidence that this influence can be predicted to some degree. In particular the perceived prominence of a node tends to increase when the node is more central in the arrangement. Also, the number of perceived groups changes when nodes are spatially clustered to hide or highlight a clique. The spatial arrangement that has the least variation between nodes in Euclidean features, the circle, also leads to the least perceived variation in the structural properties of the nodes.

However there do appear to be limits to the extent to which viewers' judgments can be altered by the graph layout. Perceptions of the most prominent nodes are not significantly affected by the spatial arrangements used in this study. We conjecture that nodes with high structural prominence are less affected by spatial positioning than others, although the distinction between the most prominent nodes and other nodes may be less marked in a larger, more complex graph.

Interestingly, the 'best' spatial arrangement for a social network may often depend on the information that the arrangement is intended to convey. In this study, the arrangement which led to the most accurate perceptions of the number of groups was different from the one that led to the most accurate perception of relative prominence. However, the circle never performed well because it appeared to hide the structural differences between the nodes.

Further work is needed to verify the generality of the results of this study, particularly with larger network structures, and with a greater number of arrangements in which Euclidean properties can be systematically varied. But even in this simple case we see that judgments of stark structural attributes are easily manipulated by spatial arrangement. It is our hope that this study provides a first step toward understanding how these biases in judgment occur, and how they can be reduced. 


\section{Appendix A}

The following ten tables show pair-wise comparisons for the five focal nodes. For both prominence and bridging, ordering that matches the structural measures is $A=B>$ $C>D=E$. For any two nodes, $x_{i}$ and $x_{j}$, where $i<j$, we can count the number of respondents who judge $x_{i}$ to be greater than, equal to, or less than $x_{j}$. For each pair $\left(x_{i}, x_{j}\right)$ there is a correct ordering. We highlight the correct ordering in boldface.

Table A.1: Arrangement 1 prominence

\begin{tabular}{|c|c|c|c|c|c|}
\hline & A & B & $\mathrm{C}$ & $\mathrm{D}$ & $\mathrm{E}$ \\
\hline A & $X$ & $\begin{array}{l}>11(25.0) \\
=21(47.7) \\
<12(27.3)\end{array}$ & $\begin{array}{l}>27(61.4) \\
=8(18.2) \\
<9(20.5)\end{array}$ & $\begin{array}{l}>25(55.6) \\
=4(8.9) \\
<16(35.6)\end{array}$ & $\begin{array}{l}>26(57.8) \\
=3(6.7) \\
<16(35.6)\end{array}$ \\
\hline B & & $X$ & $\begin{array}{l}>27(61.4) \\
=10(22.7) \\
<7(15.9)\end{array}$ & $\begin{array}{l}>21(47.7) \\
=8(18.2) \\
<15(34.1)\end{array}$ & $\begin{array}{l}>23(52.3) \\
=6(13.6) \\
<15(13.4)\end{array}$ \\
\hline $\mathrm{C}$ & & & $X$ & $\begin{array}{l}>19(43.2) \\
=7(15.9) \\
<18(40.9)\end{array}$ & $\begin{array}{l}>20(45.5) \\
=6(13.6) \\
<18(40.9)\end{array}$ \\
\hline D & & & & $X$ & $\begin{array}{l}>3(6.7) \\
=39(86.7) \\
<3(6.7)\end{array}$ \\
\hline $\mathrm{E}$ & & & & & $X$ \\
\hline
\end{tabular}

Table A.2: Arrangement 2 prominence

\begin{tabular}{|c|c|c|c|c|c|}
\hline & A & B & $\mathrm{C}$ & $\mathrm{D}$ & $\mathrm{E}$ \\
\hline A & $X$ & $\begin{array}{l}>4(8.2) \\
=44(89.8) \\
<1(2.0)\end{array}$ & $\begin{array}{l}>\mathbf{3 4}(\mathbf{7 0 . 8 )} \\
=13(27.1) \\
<1(2.1)\end{array}$ & $\begin{array}{l}>\mathbf{3 8}(79.2) \\
=8(16.7) \\
<2(4.2)\end{array}$ & $\begin{array}{l}>39(\mathbf{7 9 . 6 )} \\
=10(20.4) \\
<0(0.0)\end{array}$ \\
\hline B & & $X$ & $\begin{array}{l}>32(66.7) \\
=15(31.3) \\
<1(2.1)\end{array}$ & $\begin{array}{l}>\mathbf{3 6}(\mathbf{7 5 . 0}) \\
=8(16.7) \\
<4(8.3)\end{array}$ & $\begin{array}{l}>37(75.5) \\
=9(18.4) \\
<3(6.1)\end{array}$ \\
\hline $\mathrm{C}$ & & & $X$ & $\begin{array}{l}>\mathbf{3 4}(\mathbf{7 0 . 8 )} \\
=10(20.8) \\
<4(8.3)\end{array}$ & $\begin{array}{l}>\mathbf{3 4}(\mathbf{7 0 . 8}) \\
=10(20.8) \\
<4(8.3)\end{array}$ \\
\hline D & & & & $X$ & $\begin{array}{l}>2(4.2) \\
=43(89.6) \\
<3(6.3)\end{array}$ \\
\hline $\mathrm{E}$ & & & & & $X$ \\
\hline
\end{tabular}


Table A.3: Arrangement 3 prominence

\begin{tabular}{|c|c|c|c|c|c|}
\hline & A & B & $\mathrm{C}$ & D & $E$ \\
\hline $\mathrm{A}$ & $X$ & $\begin{array}{l}>9(20.5) \\
=26(59.1) \\
<9(20.5)\end{array}$ & $\begin{array}{l}>24(54.5) \\
=11(25.6) \\
<9(20.5)\end{array}$ & $\begin{array}{l}>\mathbf{3 0}(68.2) \\
=9(20.5) \\
<5(11.4)\end{array}$ & $\begin{array}{l}>\mathbf{2 9}(\mathbf{6 5 . 9 )} \\
=10(22.7) \\
<5(11.4)\end{array}$ \\
\hline B & & $X$ & $\begin{array}{l}>22(50.0) \\
=14(31.8) \\
<8(18.2)\end{array}$ & $\begin{array}{l}>29(65.9) \\
=10(22.7) \\
<5(11.4)\end{array}$ & $\begin{array}{l}>\mathbf{2 8}(\mathbf{6 3 . 6}) \\
=11(25.0) \\
<5(11.4)\end{array}$ \\
\hline C & & & $X$ & $\begin{array}{l}>27(61.4) \\
=11(25.0) \\
<6(13.6)\end{array}$ & $\begin{array}{l}>\mathbf{2 6}(\mathbf{5 9 . 1}) \\
=12(27.3) \\
<6(13.6)\end{array}$ \\
\hline D & & & & $X$ & $\begin{array}{l}>9(20.5) \\
=\mathbf{2 7}(61.4) \\
<8(18.2)\end{array}$ \\
\hline$E$ & & & & & $X$ \\
\hline
\end{tabular}

Table A.4: Arrangement 4 prominence

\begin{tabular}{|c|c|c|c|c|c|}
\hline & A & B & $\mathrm{C}$ & D & E \\
\hline A & $X$ & $\begin{array}{l}>2(4.5) \\
=40(90.9) \\
<2(4.5)\end{array}$ & $\begin{array}{l}>25(\mathbf{5 9 . 9 )} \\
=8(19.0) \\
<9(21.4)\end{array}$ & $\begin{array}{l}>\mathbf{2 6}(\mathbf{6 1 . 9}) \\
=7(16.7) \\
<9(21.4)\end{array}$ & $\begin{array}{l}>27(61.4) \\
=12(27.3) \\
<5(11.4)\end{array}$ \\
\hline B & & $X$ & $\begin{array}{l}>\mathbf{2 5}(\mathbf{5 9 . 9 )} \\
=8(19.0) \\
<9(21.4)\end{array}$ & $\begin{array}{l}>\mathbf{2 5}(\mathbf{5 9 . 5}) \\
=7(16.7) \\
<10(23.8)\end{array}$ & $\begin{array}{l}>\mathbf{2 6}(\mathbf{5 9 . 1}) \\
=12(27.3) \\
<6(13.6)\end{array}$ \\
\hline C & & & $X$ & $\begin{array}{l}>\mathbf{2 1}(\mathbf{5 0 . 0}) \\
=11(26.2) \\
<10(23.8)\end{array}$ & $\begin{array}{l}>20(47.6) \\
=10(23.8) \\
<12(28.6)\end{array}$ \\
\hline D & & & & $X$ & $\begin{array}{l}>8(19.0) \\
=\mathbf{3 0}(\mathbf{7 1 . 4}) \\
<4(9.5)\end{array}$ \\
\hline E & & & & & $X$ \\
\hline
\end{tabular}


Table A.5: Arrangement 5 prominence

\begin{tabular}{|c|c|c|c|c|c|}
\hline & A & B & $\mathrm{C}$ & $\mathrm{D}$ & $\mathrm{E}$ \\
\hline $\mathrm{A}$ & $X$ & $\begin{array}{l}>15(34.1) \\
=18(40.9) \\
<11(25.0)\end{array}$ & $\begin{array}{l}>25(56.8) \\
=13(29.5) \\
<6(13.6)\end{array}$ & $\begin{array}{l}>25(56.8) \\
=16(36.4) \\
<3(6.8)\end{array}$ & $\begin{array}{l}>26(59.1) \\
=11(25.0) \\
<7(15.9)\end{array}$ \\
\hline B & & $X$ & $\begin{array}{l}>23(\mathbf{5 1 . 5}) \\
=13(28.9) \\
<9(20.0)\end{array}$ & $\begin{array}{l}>27(61.4) \\
=12(27.3) \\
<5(11.4)\end{array}$ & $\begin{array}{l}>27(61.4) \\
=10(22.7) \\
<7(15.9)\end{array}$ \\
\hline $\mathrm{C}$ & & & $X$ & $\begin{array}{l}>23(52.3) \\
=14(31.8) \\
<7(15.9)\end{array}$ & $\begin{array}{l}>24(54.5) \\
=14(31.8) \\
<6(13.6)\end{array}$ \\
\hline $\mathrm{D}$ & & & & $X$ & $\begin{array}{l}>10(22.7) \\
=23(52.3) \\
<11(25.0)\end{array}$ \\
\hline $\mathrm{E}$ & & & & & $X$ \\
\hline
\end{tabular}

Table A.6: Arrangement 1 bridging

\begin{tabular}{|c|c|c|c|c|c|}
\hline & A & B & $\mathrm{C}$ & D & $\mathrm{E}$ \\
\hline $\mathrm{A}$ & $X$ & $\begin{array}{l}>7(15.9) \\
=31(70.5) \\
<6(13.6)\end{array}$ & $\begin{array}{l}>32(72.7) \\
=5(11.4) \\
<7(15.9)\end{array}$ & $\begin{array}{l}>38(86.4) \\
=3(6.8) \\
<3(6.8)\end{array}$ & $\begin{array}{l}>37(84.1) \\
=3(6.8) \\
<4(9.1)\end{array}$ \\
\hline B & & $X$ & $\begin{array}{l}>33(\mathbf{7 5 . 0}) \\
=4(9.1) \\
<7(15.9)\end{array}$ & $\begin{array}{l}>38(84.4) \\
=4(8.9) \\
<3(6.7)\end{array}$ & $\begin{array}{l}>37(82.2) \\
=4(8.9) \\
<4(8.9)\end{array}$ \\
\hline C & & & $X$ & $\begin{array}{l}>35(79.5) \\
=4(9.1) \\
<5(11.4)\end{array}$ & $\begin{array}{l}>35(79.5) \\
=3(6.8) \\
<6(13.6)\end{array}$ \\
\hline $\mathrm{D}$ & & & & $X$ & $\begin{array}{l}>1(2.2) \\
=41(91.1) \\
<3(6.7)\end{array}$ \\
\hline $\mathrm{E}$ & & & & & $X$ \\
\hline
\end{tabular}


Table A.7: Arrangement 2 bridging

\begin{tabular}{|c|c|c|c|c|c|}
\hline & A & B & $\mathrm{C}$ & $\mathrm{D}$ & $\mathrm{E}$ \\
\hline $\mathrm{A}$ & $X$ & $\begin{array}{l}>2(4.1) \\
=46(93.9) \\
<1(2.0)\end{array}$ & $\begin{array}{l}>38(77.6) \\
=9(18.4) \\
<2(4.1)\end{array}$ & $\begin{array}{l}>47(95.9) \\
=1(2.0) \\
<1(2.0)\end{array}$ & $\begin{array}{l}>47(95.9) \\
=2(4.1) \\
<0(0.0)\end{array}$ \\
\hline B & & $X$ & $\begin{array}{l}>39(\mathbf{7 9 . 6}) \\
=7(14.3) \\
<3(6.1)\end{array}$ & $\begin{array}{l}>45(91.8) \\
=1(2.0) \\
<3(6.1)\end{array}$ & $\begin{array}{l}>45(91.8) \\
=2(4.1) \\
<2(4.1)\end{array}$ \\
\hline $\mathrm{C}$ & & & $X$ & $\begin{array}{l}>42(85.7) \\
=6(12.2) \\
<1(2.1)\end{array}$ & $\begin{array}{l}>42(85.7) \\
=6(12.2) \\
<1(2.1)\end{array}$ \\
\hline $\mathrm{D}$ & & & & $X$ & $\begin{array}{l}>4(8.2) \\
=44(89.8) \\
<1(2.0)\end{array}$ \\
\hline $\mathrm{E}$ & & & & & $X$ \\
\hline
\end{tabular}

Table A.8: Arrangement 3 bridging

\begin{tabular}{|c|c|c|c|c|c|}
\hline & A & B & $\mathrm{C}$ & $\mathrm{D}$ & $\mathrm{E}$ \\
\hline A & $X$ & $\begin{array}{l}>9(20.5) \\
=25(56.8) \\
<10(22.7)\end{array}$ & $\begin{array}{l}>26(57.8) \\
=8(17.8) \\
<11(24.4)\end{array}$ & $\begin{array}{l}>\mathbf{3 8}(\mathbf{8 6 . 4 )} \\
=4(9.1) \\
<2(4.5)\end{array}$ & $\begin{array}{l}>38(84.4) \\
=3(6.8) \\
<4(8.7)\end{array}$ \\
\hline B & & $X$ & $\begin{array}{l}>27(\mathbf{6 1 . 4}) \\
=6(13.6) \\
<11(25.0)\end{array}$ & $\begin{array}{l}>38(\mathbf{8 8 . 8 )} \\
=4(9.3) \\
<1(2.3)\end{array}$ & $\begin{array}{l}>37(84.1) \\
=5(11.1) \\
<3(6.7)\end{array}$ \\
\hline $\mathrm{C}$ & & & $X$ & $\begin{array}{l}>\mathbf{3 9}(\mathbf{8 8 . 6 )} \\
=2(4.5) \\
<3(6.8)\end{array}$ & $\begin{array}{l}>37(82.2) \\
=5(11.1) \\
<3(6.7)\end{array}$ \\
\hline $\mathrm{D}$ & & & & $X$ & $\begin{array}{l}>10(22.7) \\
=24(54.5) \\
<10(22.7)\end{array}$ \\
\hline$E$ & & & & & $X$ \\
\hline
\end{tabular}


Table A.9: Arrangement 4 bridging

\begin{tabular}{|c|c|c|c|c|c|}
\hline & $\mathrm{A}$ & B & $\mathrm{C}$ & D & $\mathrm{E}$ \\
\hline A & $X$ & $\begin{array}{l}>1(2.3) \\
=\mathbf{3 8}(\mathbf{8 9 . 4 )} \\
<4(9.3)\end{array}$ & $\begin{array}{l}>\mathbf{2 7}(\mathbf{6 2 . 8}) \\
=3(7.0) \\
<13(30.2)\end{array}$ & $\begin{array}{l}>32(76.2) \\
=4(9.5) \\
<6(14.3)\end{array}$ & $\begin{array}{l}>34(79.1) \\
=4(9.3) \\
<5(11.6)\end{array}$ \\
\hline B & & $X$ & $\begin{array}{l}>\mathbf{2 8}(\mathbf{6 5 . 1}) \\
=3(7.0) \\
<12(27.9)\end{array}$ & $\begin{array}{l}>\mathbf{3 4}(\mathbf{7 9 . 1}) \\
=4(9.3) \\
<5(11.6)\end{array}$ & $\begin{array}{l}>35(79.5) \\
=7(15.9) \\
<2(4.5)\end{array}$ \\
\hline C & & & $X$ & $\begin{array}{l}>35(83.3) \\
=3(7.1) \\
<4(9.5)\end{array}$ & $\begin{array}{l}>37(\mathbf{8 6 . 0}) \\
=2(4.7) \\
<4(9.3)\end{array}$ \\
\hline $\mathrm{D}$ & & & & $\mathrm{X}$ & $\begin{array}{l}>7(16.3) \\
=33(76.7) \\
<3(7.0)\end{array}$ \\
\hline$E$ & & & & & $X$ \\
\hline
\end{tabular}

Table A.10: Arrangement 5 bridging

\begin{tabular}{|c|c|c|c|c|c|}
\hline & A & B & $\mathrm{C}$ & D & $\mathrm{E}$ \\
\hline A & $X$ & $\begin{array}{l}>13(30.2) \\
=20(46.5) \\
<10(23.3)\end{array}$ & $\begin{array}{l}>\mathbf{2 5}(\mathbf{5 8 . 1}) \\
=13(30.2) \\
<5(11.6)\end{array}$ & $\begin{array}{l}>\mathbf{2 4}(\mathbf{5 5 . 8}) \\
=14(32.6) \\
<5(11.6)\end{array}$ & $\begin{array}{l}>23(55.5) \\
=17(39.5) \\
<3(7.0)\end{array}$ \\
\hline B & & $X$ & $\begin{array}{l}>21(47.7) \\
=16(36.4) \\
<7(15.9)\end{array}$ & $\begin{array}{l}>23(52.3) \\
=13(29.5) \\
<8(18.2)\end{array}$ & $\begin{array}{l}>26(59.1) \\
=11(25.0) \\
<7(15.9)\end{array}$ \\
\hline $\mathrm{C}$ & & & $X$ & $\begin{array}{l}>17(38.6) \\
=16(36.4) \\
<11(25.0)\end{array}$ & $\begin{array}{l}>22(50.0) \\
=13(29.5) \\
<9(20.5)\end{array}$ \\
\hline D & & & & $\mathrm{X}$ & $\begin{array}{l}>12(27.3) \\
=\mathbf{2 2}(\mathbf{5 0 . 0}) \\
<10(22.7)\end{array}$ \\
\hline$E$ & & & & & $X$ \\
\hline
\end{tabular}

\section{References}

Batini, C., L. Furlani and E. Nardelli, 1985, What is a good diagram? A pragmatic approach, in: 4th International Conference on the Entity Relationship Approach, Chicago.

Borgatti, S. and M. Everett, 1992, Regular block models of multiway, multimode matrices, Social Networks $14,91-120$. 
Di Battista, G., P. Eades, R. Tamassia and I. Tollis, 1994, Algorithms for drawing graphs: An annotated bibliography, Computational geometry: Theory and applications, 4, 235-282.

Ding, C. and P. Mateti, 1990, A framework for the automated drawing of data structure diagrams, IEE Transactions on Software Engineering 16, 543-557.

Freeman, L.C., 1978, Centrality in social networks conceptual clarification, Social Networks 1, 215-239.

Harary, F., 1969, Graph theory (Addison-Wesley, Reading, MA).

Larkin, J. and H. Simon, 1987, Why a diagram is (sometimes) worth ten thousand words, Cognitive Science 11, 65-99.

Moreno, J.L., 1953, Who shall survive? (Beacon House, New York).

Poulton, E.C., 1982, Influential companions: Effects of one strategy on another in the within-subjects designs of cognitive psychology, Psychological Bulletin 91, 673-690.

Scott, J., 1991, Social network analysis: A handbook (Sage, London). 